\title{
Guest editors' introduction: special issue on mining and learning with graphs
}

\author{
Thomas Gärtner • Gemma C. Garriga
}

Received: 5 January 2009 / Revised: 5 January 2009 / Accepted: 9 January 2009 /

Published online: 17 January 2009

Springer Science+Business Media, LLC 2009

Research in machine learning and data mining acknowledges more and more the fact that most data occurring in real world problems is inherently structured. Graphs and particular subclasses of graphs are commonly used representations for such structured data. They are present in important application areas ranging from biology and chemistry to the World Wide Web and beyond. Following the ever increasing number of successful workshops on topics like mining and learning with graphs and motivated by the ever increasing amount of high quality submissions to these workshops, this special issue gathers some of the progress in the field over the recent years.

One of the major trends has been to investigate how the success of kernel methods on data which is readily embedded in a Euclidean space, can be carried over to structured data. In this spirit, the paper "Graph kernels based on tree patterns for molecules" by Pierre Mahé and Jean-Philippe Vert investigates kernel functions based on co-occurrence of particular subtree patterns in graphs. They extend and use these kernels for toxicity and anti-cancer activity prediction with support vector machines on graphs representing the 2D structure of molecules.

The paper "On the properties of von Neumann kernels for link analysis" by Masashi Shimbo, Takahiko Ito, Daichi Mochihashi, and Yuji Matsumoto investigates the relationship between link analysis measures and kernel functions defined on the vertices of a single huge graph. Having identified common issues of both these kernels and these link analysis measures, the authors proceed by proposing a modified kernel and demonstrate its effectiveness by analysing a citation network of scientific papers.

T. Gärtner (凶)

Knowledge Discovery, Fraunhofer Institute Intelligent Analysis and Information Systems IAIS,

Schloss Birlinghoven, 53754 Sankt Augustin, Germany

e-mail: thomas.gaertner@iais.fraunhofer.de

G.C. Garriga

Helsinki Institute for Information Technology, Helsinki University of Technology, Helsinki, Finland e-mail: gemma.garriga@ hut.fi 
While these papers were more machine learning than data mining oriented, the paper "gBoost: a mathematical programming approach to graph classification and regression" by Hiroto Saigo, Sebastian Nowozin, Tadashi Kadowaki, Taku Kudo, and Koji Tsuda demonstrates the potential benefit of methods that combine the best from both worlds. They propose a mathematical programming boosting method that progressively collects informative patterns and builds a classifier based on occurrence of these patterns in a set of graphs. With such an approach, a wide range of machine learning problems can be solved without modifying the pattern search algorithm.

The paper "Mining probabilistic automata: a statistical view of sequential pattern mining" by Stéphanie Jacquemont, François Jacquenet, and Marc Sebban focuses on sequential data mining and use probabilistic automata as compact and generalised representations of the original sequences. The authors further investigate how to integrate statistical constraints and how the probabilistic automata help to ensure the preservation of privacy in personalised data.

Last but not least, the paper "An efficient algorithm for learning to rank from preference graph" by Tapio Pahikkala, Evgeni Tsivtsivadze, Antti Airola, Jouni Järvinen, and Jorma Boberg investigates the problem of inducing a total order from a given directed preference graph, ideally a partial order. Their proposed solution is based on a regularised least squares formulation and they demonstrate that several advanced techniques for regularised least squares regression do carry over to the ranking case.

Summing up, we believe that these papers provide a good overview of some of the most important trends in mining and learning with graphs. We wish to thank all the people who contributed to this special issue in one or the other way. Many thanks to all the reviewers who gave high quality feedback on the papers, many thanks to the people at Springer for their help, many thanks to Foster Provost for his support, and most of all, many thanks to all the authors of all 31 submissions that we received for making this special issue possible. 